\title{
FM 17:
}

Advances in Stellar Physics from Asteroseismology 


\title{
Scaling of oscillation frequencies in rotating stars
}

\author{
Diego Castaneda and Robert G. Deupree \\ Saint Mary's University, Institute for Computational Astrophysics, \\ 923 Robbie Street, Halifax, NS, Canada \\ email: castaned@ap.smu.ca
}

\begin{abstract}
We have studied oscillation frequencies of two-dimensional uniformly rotating zeroage main sequence stellar models in the delta Scuti mass range. We identified $370 \mathrm{p}$ and $\mathrm{g}$ axisymmetric modes for non-rotating models and then traced their evolution as the rotational velocity was increased. For each mass we considered a rotation sequence of ten models, with the largest rotation rate being about $200 \mathrm{~km} \mathrm{~s}^{-1}$. We constrained the models to have the same surface shape, which can be characterized for uniform rotation by the ratio between the polar and the equatorial radii. We find that scaling relationships exist among the oscillation frequencies calculated for models with the same shape. For p modes, this scaling closely follows the period root-mean-density relation found in spherical stars. The g modes also scale between models of the same shape, with the scaling reflecting the change in properties outside the convective core as the stellar mass increases. These scaling relationships can be particularly useful in finding specific stellar models to match the oscillation frequencies of individual stars.
\end{abstract}

\section{Introduction}

The use of theoretical models to match observed oscillation frequencies of rotating stars can be a lengthy and cumbersome process that in the end rarely ever leads to certain conclusions. A number of challenges stand in the way of calculating these frequencies for this type of stars, and most of them arise because rotation breaks the spherical symmetry in the star. When this happens the horizontal and radial components of the eigenfunctions in a linear pulsation analysis no longer decouple as they do for a non-rotating star. This means that the latitudinal component of the eigenfunction can no longer be given by a single Legendre polynomial, but must be regarded as a sum of Legendre polynomials. This has led to two dimensional solutions to the eigenvalue problem (e.g., Clement 1998, Yoshida \& Eriguchi 2001, Espinosa 2004, Lignieres et. al. 2001, Lovekin \& Deupree 2008, Lovekin et. al. 2009) but further studies are required to fully understand how to properly calculate and compare theoretical oscillation frequencies of rotating stars with observations.

In cases where there are reasonable rotating models to calculate theoretical oscillation frequencies for comparison to those observed on actual rotating stars, it is unlikely that any of these models will match what is observed as closely as desired. Corrections must be made to the models in question to improve the agreement between the observed and the oscillation frequencies of the models. This can be most easily performed if there are scaling relations between the oscillation frequencies of two models, much like the period-root mean density relation for non-rotating stars, to guide the correction process. It is often assumed that the the period-root mean density relation can be applied in the same way for rotating stars as its used for the non-rotating cases, but this remains to be proven. In addition, g modes cannot be expected to follow this same relationship, and it is of interest to see if a scaling relation exists for g modes. We present results that 
Table 1. Rotating models considered

\begin{tabular}{|c|c|c|c|c|c|}
\hline & & Mass & $\left(\mathrm{M}_{\odot}\right)$ & & \\
\hline & 1.875 & 2.000 & 2.250 & 2.500 & \\
\hline Shape $\left(R_{\text {pole }} / R_{\text {eq }}\right)$ & & locities & $(\mathrm{km} \mathrm{s}$ & $-1)$ & $T_{\text {eff }, \text { pole }} / T_{\text {eff,eq }}$ \\
\hline 1.000 & 0.0 & 0.0 & 0.0 & 0.0 & 1.000 \\
\hline 0.997 & 35.0 & 36.0 & 36.0 & 37.5 & 1.003 \\
\hline 0.991 & 62.0 & 63.0 & 65.0 & 67.0 & 1.009 \\
\hline 0.985 & 83.0 & 84.0 & 87.0 & 89.0 & 1.015 \\
\hline 0.976 & 105.0 & 106.0 & 109.0 & 111.0 & 1.024 \\
\hline 0.966 & 125.0 & 127.0 & 131.0 & 134.0 & 1.034 \\
\hline 0.954 & 146.0 & 148.0 & 152.0 & 156.0 & 1.047 \\
\hline 0.940 & 165.0 & 168.0 & 173.0 & 178.0 & 1.064 \\
\hline 0.925 & 187.0 & 190.0 & 195.0 & 200.0 & 1.079 \\
\hline 0.907 & 207.0 & 211.0 & 217.0 & 222.0 & 1.098 \\
\hline
\end{tabular}

suggest that such scaling relationships exist for rotating stars but some considerations need to be made before they can be applied.

\section{Calculation of oscillation frequencies}

The models considered are a subset of the models computed by Deupree (2011a). They are uniformly rotating, zero-age main sequence (ZAMS) models of solar metallicity. The masses selected cover the $\delta$ Scuti range: $1.875 M_{\odot}$ to $2.5 M_{\odot}$. Finally, for each mass we picked ten surface equatorial rotational velocities, keeping $R_{\mathrm{pole}} / R_{\mathrm{eq}}$ of the model constant between the different masses at every step in rotational velocity.

For each model we calculated $370 \mathrm{p}$ and $\mathrm{g}$ axisymmetric oscillation modes using the linear, adiabatic, non-radial pulsation code developed by Clement (1998). This particular code can express the latitudinal part of the eigenfunctions of each mode as a sum of up to the eight spherical harmonics we use here. We characterize each mode with the value of $\ell$ associated with the mode in the non-rotating case. While tracking the individual modes as a function of rotation becomes difficult at higher rotation rates, identifying the same mode in two models with the same surface shape but different masses is generally quite straightforward.

\section{Results}

We present the ratio of the frequencies for modes with $\ell \leqslant 6$ for models with masses $=$ 2 and $2.25 \mathrm{M}_{\odot}$ in Figure 1 (left). It is clear that the p modes follow the period root mean density relation (given by the horizontal line) relatively well. The g mode frequencies at low frequency have the ratio determined from the asymptotic relation based on the integration of the Brunt-Väisälä frequency (Tassoul 1980). The low latitudinal order g modes have frequency ratios between those of the $\mathrm{p}$ mode and asymptotic $\mathrm{g}$ mode ratios.

It is often assumed that the period-root mean density relation remains true in rotating stars. This is confirmed in Figure 1 (right), which shows the frequency ratio for the same masses for reasonably rapid rotation. The g mode frequency ratio at low frequency is also very close to that of the non-rotating models because the $g$ mode frequencies are determined in the interior regions outside the convective core where the rotational effects are small. The individual modes which are most affected are the low radial order $\mathrm{g}$ modes and mixed modes. Generally speaking, higher latitudinal order modes in this region are more affected than lower ones. A number of the modes in this region show avoided 

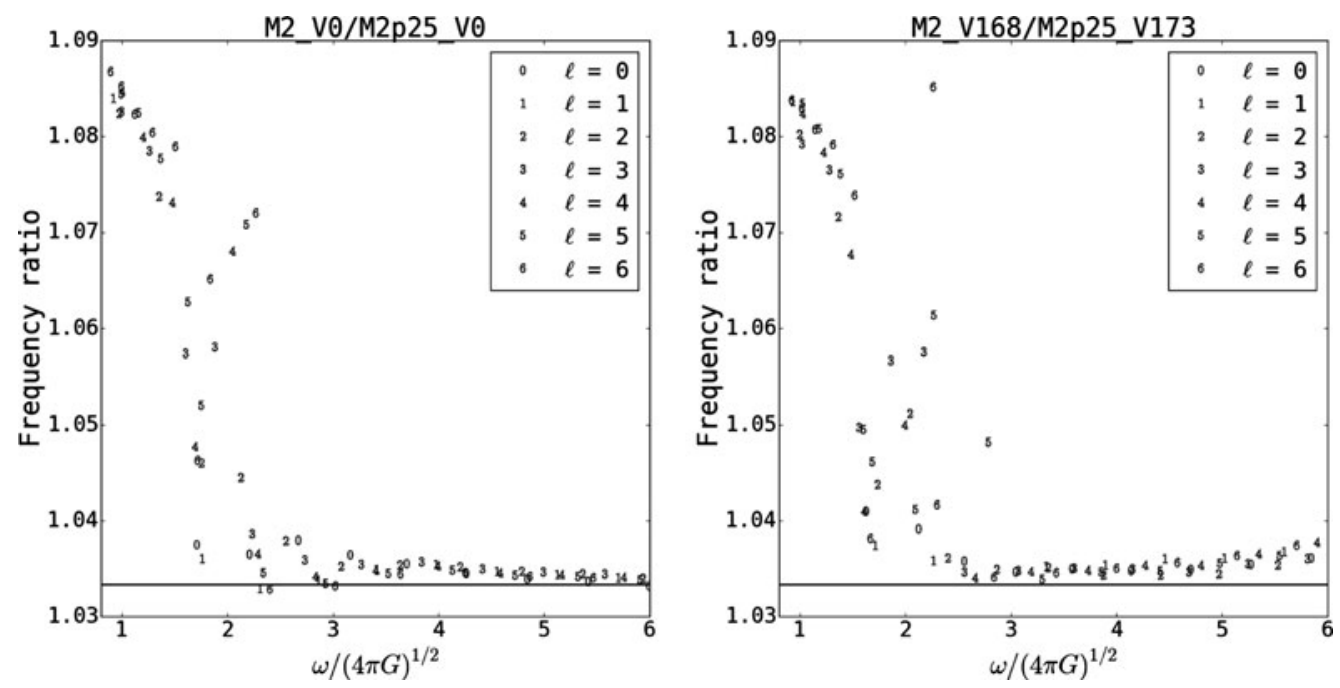

Figure 1. Ratio between frequencies of two non-rotating models (left) and two rotating models (right). The black horizontal line indicates the ratio between the root-mean densities of both models.

crossings as the rotation rate increases, and some of these modes may be misidentified, particularly when the mode in one mass has had an avoided crossing while the mode in the other mass may not yet have had it.

\section{Summary}

We have examined the variation of both $\mathrm{g}$ mode and $\mathrm{p}$ mode oscillation frequencies in intermediate mass, uniformly rotating, ZAMS stellar models. By comparing models of different masses with the same surface shape we found that the ratio of the frequencies of the same mode is relatively unaffected for the $\mathrm{p}$ modes. This is qualified by the same restriction as the period-root mean density in non-rotating stars, namely that the relation works well as long as the two models are somewhat "alike" in their internal structure (e.g., both main sequence models). The other restriction is that the two rotating models must have the same surface shape.

The $\mathrm{g}$ mode frequencies are much less affected by rotation because their frequencies are determined in the region of the model between the convective core boundary and the lower boundary of the second helium ionization region. The region closer to the convective core is probably more important, but the entire region contributes to the frequency determination. For sufficiently low frequencies, the frequency ratio of a given mode for two masses is almost independent of the mode. There is a slight trend of increasing frequency ratio with decreasing frequency as shown in Figure 1. The low order g modes and mixed modes show much greater volatility in this dense part of the frequency spectrum. This fact may be useful in mode classification if enough modes are observed covering both the $\mathrm{p}$ and $\mathrm{g}$ mode parts of the spectrum.

\section{References}

Clement, M. J. 1998, ApJS , 116, 57

Deupree, R. G. 2011, ApJ, 735, 69 
Espinosa, F., Pérez Hernández F., \& Roca Cortés T., 2004, in Danesy D., ed., SOHO 14 Helioand Asteroseismology: Towards a Golden Future Vol. 559 of ESA Special Publication, Oscillation Modes in Axially Symmetric Stars. p. 424

Lignières F., Rieutord, M., \& Reese, D. 2006, A\&A , 455, 607

Lovekin, C. C. \& Deupree, R. G. 2008, ApJ , 679, 1499

Lovekin, C. C., Deupree, R. G., \& Clement, M. J. 2009, ApJ , 693, 677

Tassoul, M. 1980, ApJS , 43, 469

Yoshida, S. \& Eriguchi, Y. 2001, MNRAS , 322, 389 